Original article

\title{
Seroprevalence of toxocariasis and its related risk factors among municipal street sweepers in Shiraz District in Fars Province, southern Iran
}

\author{
Amirhossein Erfani $^{\mathrm{a}, \mathrm{b}}$, Ali Pouryousef ${ }^{\mathrm{a}}$, Nasir Arefkhah ${ }^{\mathrm{a}}$, Reza Shahriarirad ${ }^{\mathrm{b}}$, \\ Bahador Sarkari ${ }^{\mathrm{a}, \mathrm{d}, *}$ \\ a Department of Parasitology and Mycology, School of Medicine, Shiraz University of Medical Sciences, Shiraz, Iran \\ ${ }^{\mathrm{b}}$ Student Research Committee, School of Medicine, Shiraz University of Medical Sciences, Shiraz, Iran \\ ${ }^{\mathrm{c}}$ Department of Bacteriology and Virology, School of Medicine, Shiraz University of Medical Sciences, Shiraz, Iran \\ ${ }^{\mathrm{d}}$ Basic Sciences in Infectious Diseases Research Center, Shiraz University of Medical Sciences, Shiraz, Iran
} Mohammad Rastegarian $^{\mathrm{b}}$, Ali Zeighami ${ }^{\mathrm{b}}$, Fattaneh Mikaeili ${ }^{\mathrm{a}}$, Seyed Younes Hosseini ${ }^{\mathrm{c}}$,

\section{A R T I C L E I N F O}

\section{Keywords:}

Seroprevalence

Toxocariasis

Street sweepers

Fars province

Iran

\begin{abstract}
A B S T R A C T
Background: Sweeping the street is a hazardous job that exposes the workers to soil-transmitted diseases, in particular, toxocariasis. In this cross-sectional study, the seroprevalence of Toxocara infection among street sweepers in Shiraz, southern Iran was assessed. Methods: Overall, 384 subjects, aged 24-64 years (all men), were randomly selected for the detection of antibodies to Toxocara by an enzyme-linked immunosorbent assay (ELISA). Results: Anti-Toxocara antibodies were detected in sera of 101(26.3\%) out of 384 subjects. Most of the seropositive cases were in the age group of 31-50 years, yet no significant difference was found between age and Toxocara seropositivity ( $P>0.05$ ). Out of 101 seropositive cases, 21(20.8\%) had a work experience of fewer than 5 years and 80 cases $(79.2 \%)$ had a work experience greater than or equal to 5 years. However, no significant association was observed between work experience and Toxocara seropositivity. Conclusion: The findings of the study indicate that street sweepers are at high risk of infection with Toxocara infection. Therefore, appropriate preventive measures should be taken to reduce the risk of infection in this vulnerable group.
\end{abstract}

\section{Introduction}

Human toxocariasis is a roundworm infection caused by the ingestion of Toxocara canis and Toxocara cati eggs. ${ }^{1}$ After ingestion, the eggs hatch and larvae penetrate the intestinal wall and are carried by the circulation to a wide variety of tissues and may cause complications that involve the liver, eye, brain, and other human organs. ${ }^{1}$ Most of the humans' cases who become infected with Toxocara do not have serious symptoms but some may have manifestations including ocular larva migrans (OLM) or visceral larva migrans (VLM). Signs and symptoms of OLM and VLM may include vision loss, eye inflammation or damage to the retina, fever, fatigue, coughing, wheezing, and abdominal pain., ${ }^{2,3}$ Serological surveys indicate that the prevalence of toxocariasis in less industrialized, tropical countries varies from 30 up to $80 \%{ }^{4-8}$ Seroprevalence of human toxocariasis in Iran is reported to be from 1 to $34.5 \% .^{9}$ In a study by Miladi et al. in Arak in Markazi Province, in central Iran, anti-Toxocara antibodies were detected in sera of $4.2 \%$ of the individuals referred to diagnostic laboratories. ${ }^{10}$ In another study by Momeni et al. on 397 sera samples of 2-20 years old individuals in
Urmia, in West Azerbaijan Province of Iran, 12 (3\%) of cases were seropositive for toxocariasis. ${ }^{11}$ In a study by Ebrahimi-Fard in Mazandaran province, located along the southern coast of the Caspian Sea in Iran, the seroprevalence of toxocariasis in 322 healthy adults with eosinophilia was reported to be $23.5 \% .{ }^{12}$ A study in Shiraz, capital of Fars province in southern Iran on 6-13 years old schoolchildren, revealed Toxocara infection in $25.6 \%$ of the cases. ${ }^{13}$ Although epidemiological studies and reports of toxocariasis have been done in different areas of the world, no estimate of the global disease burden exists, and major knowledge gaps still remain in the epidemiology of toxocariasis. ${ }^{3}$ Sociodemographic and environmental factors such as age, geographical region, job, poverty, ethnicity, and contact with dogs and cats appear to be associated with Toxocara seropositivity. ${ }^{14}$

Contaminated soil is an important source of infection for Toxocara for both adults as well as children. Considering the importance of awareness of the seroprevalence of toxocariasis in high-risk groups such as street sweepers who are exposed to contaminated dust and soil and are prone to acquiring toxocariasis, the current study aimed to assess the seroprevalence of toxocariasis among street sweepers in Shiraz

\footnotetext{
* Corresponding author. Department of Parasitology and Mycology, School of Medicine, Shiraz University of Medical Sciences, Shiraz, Iran.

E-mail address: sarkarib@sums.ac.ir (B. Sarkari).
} 
County, in southern Iran.

\section{Methods}

\subsection{Study area}

This study was conducted in Shiraz district, which is the capital of Fars Province in southern Iran. The population of the Shiraz is $1,869,001$ (the fifth populated city in Iran). The district is located at an altitude of $1545 \mathrm{~m}$ above the sea level at geographical coordinates of $29^{\circ} 36^{\prime} 37^{\prime \prime} \mathrm{N}$ latitude and $52^{\circ} 31^{\prime} 52^{\prime \prime} \mathrm{E}$ longitudes. The area has a relatively hot summer and moderate winter. Annual precipitation is about $204 \mathrm{~mm}$. Agriculture has always been a major part of the economy in Shiraz. Fars province (especially Shiraz) is the area where a high prevalence of human toxocariasis has been reported in children in previous studies. $^{9,13,15}$ Street sweepers are continuously exposed to contaminated soil and mixed hazardous garbage which significantly affects their occupational health. Considering that the study area is one of the tourism poles in Iran, urban cleanliness is one of its main policies. Therefore, due to the existence of about 2600 street sweepers in this area and a lack of information about the seroepidemiology of toxocariasis among these people, this group of people was selected for this study. The district included 10 municipal areas and the numbers of participants were selected based on the populations of the street sweepers in each municipal area. Fig. 1 shows the study area along with the municipal areas where the samples were collected.

\subsection{Blood sampling}

Among 2600 street sweepers in the study area, 384 cases were selected by simple random sampling in 2018. Informed written consent was obtained from all of the individuals and the study was approved by the Research Ethics Committee of Shiraz University of Medical Sciences (SUMS) (ethical code: IR.sums.med.rec.1397.581). The enrollment criteria were being a municipal street sweeper in Shiraz District, and having at least one year of work experience while the exclusion criteria were generally not feeling well, having fever and reluctant to provide the blood.

All through sampling, a data collection sheet was used to take the sociodemographic data of the subjects along with determinants concerning the Toxocara risk factors. About a $5 \mathrm{ml}$ venous blood sample was taken from each participant and transferred to the laboratory of parasitology, at SUMS. Sera were separated from the blood then kept at $-20{ }^{\circ} \mathrm{C}$ until use.

\subsection{Preparation of Toxocara antigens and detection of anti-Toxocara antibodies}

Toxocara larvae excretory-secretory antigens (TES) were prepared as described by Zibaei et al. ${ }^{16}$ Briefly, T. canis eggs were isolated from the uteri of female worms and embryonated in $2.5 \%$ formalin/ringer solution at $25{ }^{\circ} \mathrm{C}$ for 30 days. The second stage larvae were collected and cultivated in RPMI medium. Culture supernatant containing TES antigens were collected, concentrated and were kept at -20 until used in an ELISA system.

To perform the ELISA, flat-bottom 96-well microplates (Corning, USA), were coated with $5 \mu \mathrm{g} / \mathrm{mL}$ of the Toxocara ES antigens and kept overnight at $4{ }^{\circ} \mathrm{C}$. Using an automated ELISA plate washing, the plates were washed, three times, with washing buffer (PBST: 0.05\% Tween 20 in PBS). After blocking with $3 \%$ skimmed milk, diluted sera $(1 / 100$ dilution in PBST solution) were added and the plates were incubated at RT for $1.5 \mathrm{~h}$. After washing as before, horseradish peroxidase-

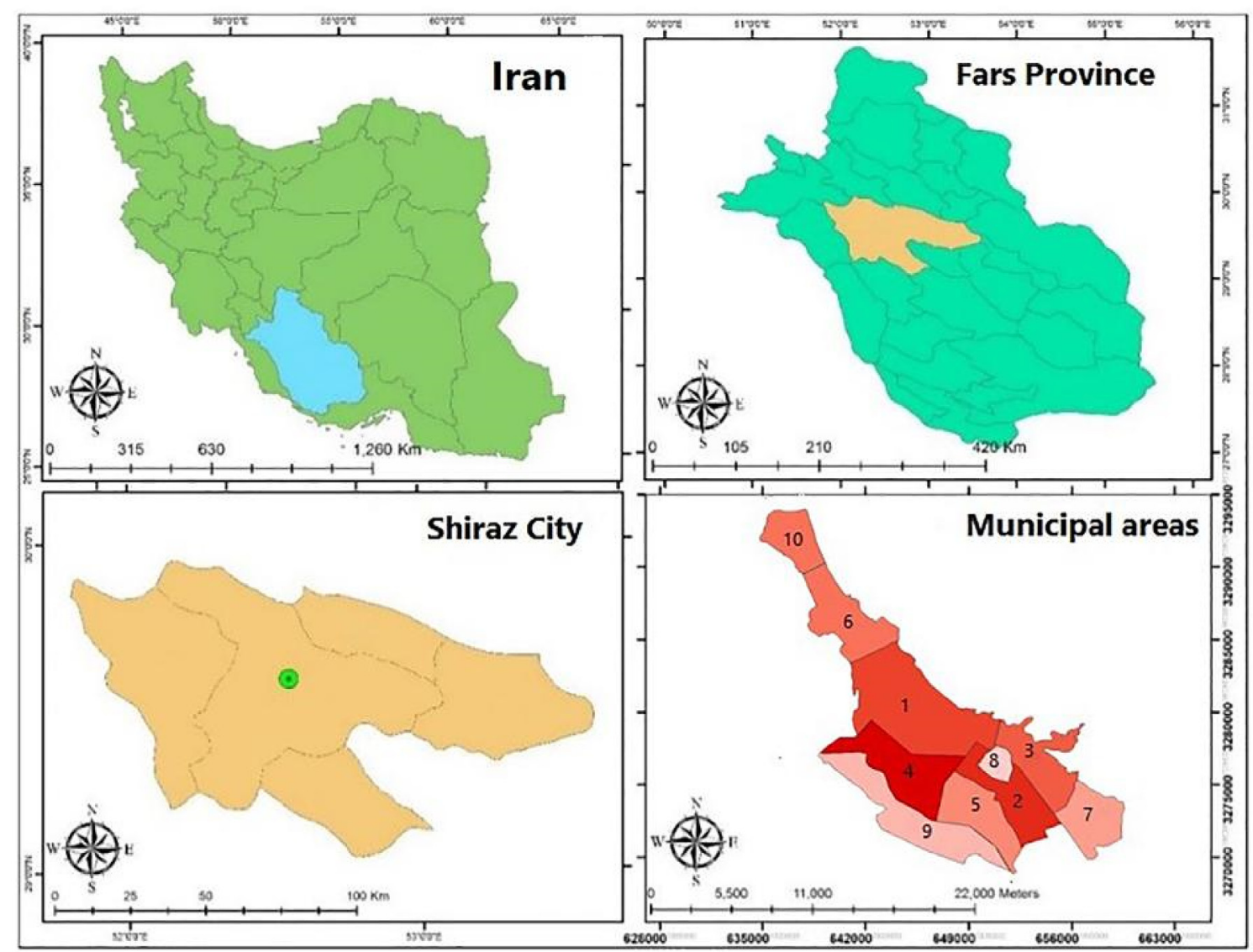

Fig. 1. Map of Iran, Fars Province and workplace (municipal areas) of street sweepers. 
conjugated goat anti-human IgG (Sigma, USA; 1/4000 in PBST) was added and after $1 \mathrm{~h}$ of incubation, and washing, OPD solution (100 $\mu \mathrm{L} /$ well of $0.4 \mathrm{mg} / \mathrm{mL} \mathrm{OPD}, 0.3 \% \mathrm{H}_{2} \mathrm{O}_{2}$ in $0.1 \mathrm{M}$ citrate buffer, $\mathrm{pH} 5$ ) was used to visualize the reaction. Using a microplate reader (ELX800, BioTek, USA), the plate was read at $490 \mathrm{~nm}$. Toxocara positive serum sample along with the negative control sera was applied in every test run and a cutoff point was calculated by the mean of negative controls OD values and two standard deviations.

\subsection{Statistical analyses}

SPSS software (version 18) was used for statistical analyses. Descriptive statistics tests were used for independent variables while a Chi-square test was used to determine the association between variables and positive results. Eventually, a value of $\mathrm{p}<0.05$ was considered as significant level.

\section{Results}

The subjects of the study were 384 street sweepers, who all were men. The mean age of the participants was $41.02( \pm 8.4)$ year. AntiToxocara antibodies were detected in sera of 101 (26.3\%) out of 384 subjects. Most of the seropositive cases were in the age group of 31-50 years. No significant association was found between age and Toxocara seropositivity $(P>0.05)$. Out of 101 seropositive cases, $21(20.8 \%)$ had work experience of fewer than 5 years and $80(79.2 \%)$ cases had a work experience greater than or equal to 5 years. However, no significant association was observed between work experience and Toxocara seropositivity. The seroprevalence of toxocariasis among those whose previous occupation was related to their current job (contact with soil and dust) was higher than those whose previous job was unrelated. Also, no associations were found between Toxocara seropositivity and having acute respiratory disease or respiratory disease along with fever and wearing the mask in the studied subjects. Table 1 shows the seroprevalence of Toxocara infection according to sociodemographic characteristics along with some of the Toxocara risk factors in studied street sweepers.

Table 1

Sociodemographic features of the street sweepers and relative seropositivity to Toxocara in Shiraz County, Fars Province, southern Iran.

\begin{tabular}{|c|c|c|c|c|c|}
\hline \multirow[t]{2}{*}{ Characteristics } & \multirow[t]{2}{*}{$\begin{array}{l}\text { Frequency } \\
\text { (No.) }\end{array}$} & \multirow[t]{2}{*}{ Percent (\%) } & \multicolumn{2}{|c|}{$\begin{array}{l}\text { Positive for anti- } \\
\text { Toxocara antibodies }\end{array}$} & \multirow[t]{2}{*}{$P$ Value } \\
\hline & & & No. & & \\
\hline \multicolumn{6}{|l|}{ Age(year) } \\
\hline $20-30$ & 43 & 11.2 & 12 & 27.9 & 0.316 \\
\hline $31-40$ & 148 & 38.6 & 38 & 25.6 & \\
\hline $41-50$ & 132 & 34.5 & 38 & 28.7 & \\
\hline$\geq 51$ & 60 & 15.7 & 13 & 21.6 & \\
\hline \multicolumn{6}{|c|}{ Contact with dust } \\
\hline Low & 51 & 13.4 & 11 & 21.5 & 0.248 \\
\hline Moderate & 114 & 29.8 & 34 & 29.8 & \\
\hline High & 119 & 31.2 & 32 & 26.8 & \\
\hline Very high & 98 & 25.7 & 24 & 24.4 & \\
\hline \multicolumn{6}{|l|}{ Previous job } \\
\hline No previous job & 311 & 81 & 81 & 26.04 & 0.573 \\
\hline Related & 52 & 13.5 & 15 & 28.8 & \\
\hline Unrelated & 21 & 5.5 & 5 & 23.8 & \\
\hline \multicolumn{6}{|c|}{ Acute pulmonary disease } \\
\hline No & 375 & 97.7 & 97 & 25.8 & 0.347 \\
\hline Yes & 9 & 2.3 & 4 & 44.4 & \\
\hline \multicolumn{6}{|l|}{ Wearing mask } \\
\hline Often & 108 & 28.1 & 26 & 24.07 & 0.390 \\
\hline Always & 276 & 71.9 & 75 & 27.1 & \\
\hline
\end{tabular}

\section{Discussion}

Toxocara infection is an important but neglected zoonosis with a relatively high prevalence in both developing and developed countries. In the current study, the seroprevalence of Toxocara infection was evaluated in street sweepers, as a high-risk group for this infection, in an area in Fars Province southern Iran, where anti-Toxocara antibodies were detected in $26.3 \%$ of the subjects. In a previous study by Sadjjadi et al. in the same area, Toxocara seroprevalence among 6-13-year-old children was found to be $25.6 \% .{ }^{13}$ In another study in the same area, anti-Toxocara antibodies were detected in sera of 5 out of $200(2.5 \%)$ patients, 3 of them with hypereosinophilia. ${ }^{17}$ The rate of infection in children in the previous study and also the municipality workers in the current study is almost the same and this is actually due to the fact that both groups are at high risk for this infection.

Differences in the Toxocara seroprevalence rates in studies conducted in southern Iran (Fars province) can be due to the variation in study population groups (sex, age, job, residence area, etc.). In a study conducted in Ilam Province, West of Iran, the seroprevalence of Toxocara antibodies in children $\leq 10$ years old, was reported to be $22 \%{ }^{18}$ Again these differences in the seroprevalence rate might be attributed to the population studied, the weather condition, health facilities, the applied serologic assays as well as the rate of soil contamination in the studied areas. The high seroprevalence of Toxocara in our study could be due to the high prevalence of Toxocara infection in dogs and cats population. ${ }^{19-21}$ The prevalence of Toxocara infections in stray dogs and cats in Fars Province, southern Iran, where the current study has been conducted, is reported to be from $3 \%$ up to $50 \%$.

It has been demonstrated that the rate of Toxocara seropositivity increases by age, as the age increases, the chances of getting infected also increase. ${ }^{2,22}$

According to the findings of a study in Egypt, the parasitic infestations were more prevalent among street sweepers (21.7\%) in comparison with the control groups. ${ }^{23}$ In another study on street sweepers in Nigeria, the frequency of parasitic infestations (intestinal parasites) was found to be quite high $(63.5 \%)$ when compared to the control counterparts. ${ }^{24}$ The high prevalence of infection in the street sweepers in these studies and also in our study highlights the fact that these groups of workers are at high risk for Toxocara infection, mainly due to their exposure to the soil and specks of dust, as the sources of contamination for Toxocara infection. Therefore, in keeping with previous studies, it can be suggested that Toxocara infection is a job-related infection. Support for this view comes from several studies including a study in Austria which showed a Toxocara seroprevalence of $44 \%$ in farmers, $27 \%$ in veterinarians, $25 \%$ in slaughterhouse staff, $17 \%$ in hunters and only $2 \%$ in the control groups. ${ }^{25}$

Population-based studies regarding Toxocara infection in healthy people indicated the common covert toxocariasis with no overt clinical signs and symptoms in these subjects. ${ }^{26-28}$ In keeping with this, in the present study, the differences in the respiratory disease between seropositive and seronegative cases were not significant. This indicated that most of the seropositive cases in our study may have covert toxocariasis. Inherited to the restrictions in most of the cross-sectional seroprevalence study, our study is not an exception and has some limitations. The sample size was not too big enough, and proper data about the exposure of the subjects to dogs and cats were not available.

\section{Conclusion}

This study represents the high prevalence of toxocariasis in street sweepers in a populated city (Shiraz) in Fars Province, southern Iran. Thus, appropriate preventive measures should be taken to reduce the risk of infection in this high-risk group of the community. 


\section{Declaration of competing interest}

None.

\section{Acknowledgments}

This study was the subject of the MD degree thesis for Amirhossein Erfani. The study was financially supported by the office of vice-chancellor for research of Shiraz University of Medical Sciences (Grant No. 97-01-01-18083).

\section{References}

1. Fialho PM, Correa CR. A systematic review of toxocariasis: a neglected but high prevalence disease in Brazil. Am J Trop Med Hyg. 2016;94:1193-1199.

2. Liu EW, Chastain HM, Shin SH, et al. Seroprevalence of antibodies to toxocara species in the United States and associated risk factors, 2011-2014. Clin Infect Dis. 2018;66:206-212.

3. Ma G, Holland CV, Wang T, et al. Human toxocariasis. Lancet Infect Dis. 2018;18:e14-e24

4. Macpherson CNL. The epidemiology and public health importance of toxocariasis: a zoonosis of global importance. Int J Parasitol. 2013;43:999-1008.

5. Schoenardie ER, Scaini CJ, Brod CS, et al. Seroprevalence of toxocara infection in children from southern Brazil. J Parasitol. 2013;99:537-539.

6. Ajayi OO, Duhlinska DD, Agwale SM, Njoku M. Frequency of human toxocariasis in jos, plateau state, Nigeria. Memórias do Inst Oswaldo Cruz. 2000;95:147-149.

7. Liao C-W, Sukati H, D'lamini P, et al. Seroprevalence of Toxocara canis infection among children in Swaziland, southern Africa. Ann Trop Med Parasitol. 2010;104:73-80.

8. Magnaval J-F, Michault A, Calon N, Charlet J-P. Epidemiology of human toxocariasis in La Reunion. Trans R Soc Trop Med Hyg. 1994;88:531-533.

9. Zibaei M, Sadjjadi SM. Trend of toxocariasis in Iran: a review on human and animal dimensions. Iran J Vet Res. 2017;18:233-242.

10. Miladi H, Didehdar M, Hajihossein R, Rafiei F, Eslamirad Z. Evaluating correlation between serum liver enzymes and toxocariasis: a case control study. Asian Pac J Trop Dis. 2016;6:460-462.

11. Momeni T, Mahami-Oskouei M, Fallah E, Safaiyan A, Mahami-Oskouei L. Latent and asymptomatic Toxocara infection among young population in Northwest Iran: the necessity of informing people as a potential health risk. Sci Tech Rep. 2016;2016:3562056.
12. Ebrahimifard SF, Fakhar M, Sharif M, Shirvani SDN. Seroprevalence of toxocara infection among adult individuals with eosinophilia in Babol, 2013. J Mazandaran University Med Sci. 2015;25:355-362.

13. Sadjjadi S, Khosravi M, Mehrabani D, Orya A. Seroprevalence of toxocara infection in school children in Shiraz, southern Iran. J Trop Pediatr. 2000;46:327-330.

14. Berrett AN, Erickson LD, Gale SD, Stone A, Brown BL, Hedges DW. Toxocara seroprevalence and associated risk factors in the United States. Am J Trop Med Hyg. 2017;97:1846-1850 (in eng).

15. Sarkari B, Alirezaei R, Layegh Gigloo A, et al. Seroprevalence and risk factors for Toxocara infection among children in a rural community in Fars province, southern Iran. Parasite Immunol. 2018;40:e12582

16. Zibaei M, Sadjjadi SM, Sarkari B, Uga S. Evaluation of Toxocara cati excretory-secretory larval antigens in serodiagnosis of human toxocariasis. J Clin Lab Anal. 2016;30:248-253.

17. Sarkari B, Lari M, Shafiei R, Sadjjadi SM. A Comparative seroprevalence study of toxocariasis in hypereosinophilic and apparently healthy individuals. Arch Pediatr Infect Dis. 2015;3:e17911.

18. Shokouhi S, Abdi J. Seroprevalence of toxocara in children from urban and rural areas of Ilam province, west Iran. Osong Public Health Res Perspect. 2018;9:101.

19. Garedaghi Y. Seroprevalence of toxocariasis in children in East-Azerbaijan province, Iran. Cukurova Med J. 2013;38:581-586.

20. Sadjjadi SM, Oryan A, Jalai AR, Mehrabani D. Prevalence and intensity of infestation with Toxocara cati in stray cats in Shiraz, Iran. Vet Arh. 2001;71:149-157.

21. Zibaei M, Sadjjadi SM, Sarkari B. Prevalence of Toxocara cati and other intestinal helminths in stray cats in Shiraz, Iran. Trop Biomed. 2007;24:39-43.

22. Cong $\mathrm{W}$, Zhang $\mathrm{X}-\mathrm{X}$, Zhou N, et al. Toxocara seroprevalence among clinically healthy individuals, pregnant women and psychiatric patients and associated risk factors in Shandong Province, Eastern China. PLoS Neglected Trop Dis. 2014;8:e3082.

23. Ewiss AA, Mohamed ES, Rahma MA, Hifnawy TM, Arafa AE. Occupational healthrelated morbidities among street sweepers and waste collectors at Beni-Suef, Egypt. Egyptian J Occup Med. 2013;37(1):79-94.

24. Mba OO. Intestinal parasites among waste-handlers in jos metropolitan area of plateau state. Nigeria. Sahel Med J. 2004;7:13-17.

25. Deutz A, Fuchs K, Auer H, Kerbl U, Aspöck H, Köfer J. Toxocara-infestations in Austria: a study on the risk of infection of farmers, slaughterhouse staff, hunters and veterinarians. Parasitol Res. 2005;97:390-394.

26. Uhlíková M, Hübner J. Seroprevalence of toxocara canis infection in Czech Republic Cent Eur J Publ Health. 1998;6:195-198 1998.

27. Abo-Shehada M, Sharif L, El-Sukhon S, Abuharfeil N, Atmeh R. Seroprevalence of Toxocara canis antibodies in humans in northern Jordan. $J$ Helminthol. 1992;66:75-78.

28. Fenoy S, Cuellar C, Guillen J. Seroprevalence of toxocariasis in children and adults in Madrid and Tenerife, Spain. J Helminthol. 1996;70:109-113. 